\title{
Serum tau fragments predict return to play in concussed professional ice hockey players
}

Shahim, $\mathrm{P}^{1}$, MD, Linemann, $\mathrm{T}^{2}$, Inekci, $\mathrm{D}^{2}$, Karsdal, $\mathrm{MA}^{2}$, Blennow, $\mathrm{K}^{1}$, MD, $\mathrm{PhD}$, Tegner, $\mathrm{Y}^{3}$, $\mathrm{MD}, \mathrm{PhD}$, Zetterberg, $\mathrm{H}^{1,4}$, MD, PhD, Henriksen $\mathrm{K}^{2}$, $\mathrm{PhD}$

${ }^{1}$ Clinical Neurochemistry Laboratory, Institute of Neuroscience and Physiology, the Sahlgrenska Academy at the University of Gothenburg, Mölndal, Sweden.

${ }^{2}$ Biomarkers \& Research, Nordic Bioscience, Herlev, Denmark.

${ }^{3}$ Division of Medical Sciences, Department of Health Sciences, Luleå University of Technology, Luleå, Sweden.

${ }^{4}$ UCL Institute of Neurology, Queen Square, London WC1N 3BG, United Kingdom.

\section{Corresponding author:}

Kim Henriksen

Nordic Bioscience A/S, Herlev Hovedgade 207, DK-2730, Denmark

Phone: $\quad+4544525219$

Fax: $\quad+4544525251$

Email: $\quad$ kh@ nordicbioscience.com

Short title: Serum tau fragments in concussed professional ice hockey players

Keywords: Sports-related concussion, serum biomarkers, tau fragments, ice hockey 


\section{Abstract (<250 words)}

Introduction: The diagnosis of sports-related concussion is mainly based on subjective clinical symptoms and neuropsychological tests. Thus, reliable brain injury biomarkers to assess when it is safe to return to play are highly desirable. The overall objective of this study was to evaluate the utility of two newly described tau fragments for diagnosis and prognosis of sports-related concussions

Materials and Methods: This multicenter prospective cohort study involved all 12 teams of the top professional ice hockey league in Sweden. A total of 288 players consented to participate in the study. Thirty-five players sustained concussions, of whom 28 underwent repeated blood samplings at 1, 12, 36, and 144 hours after the trauma, or when the player returned to play ( $7->90$ days).

Results: There was no significant increase in the levels of tau-A in post-concussion samples compared with preseason values. However, serum levels of tau-C were significantly higher in postconcussion samples compared with preseason. Further, levels of Tau-A correlated with the duration of post-concussive symptoms.

Discussion: Tau-A in serum, which is newly discovered biomarker, could be used to predict when it is safe to return to play after a sports-related concussion. 


\section{Introduction}

Concussion is common in contact sports such as ice hockey, American football, boxing and rugby. The term concussion or mild traumatic brain injury (mTBI) is used interchangeably in the medical literature. Concussion is characterized by a complex pathophysiology affecting normal brain function following exposure to excessive forces to the head (McCrory et al. 2013). Following this are short-lived neurological impairments, with symptoms such as headaches, dizziness and memory complaints. The majority of concussions resolve within 7-10 days (McCrory et al. 2013; McCrea et al. 2003; Makdissi et al. 2010) but in 10-15\% of the affected individuals, the symptoms persist for more than 10 days following injury (McCrory et al. 2013).

The standard management of sports-related concussion consists of immediate removal of players from active competition and evaluation by the team physician. Cognitive and physical rest is then recommended until the player is asymptomatic (McCrory et al. 2013;Petraglia et al. 2012). Today, due to the lack of reliable objective tools, both the diagnosis of sports-related concussion and the decision to return to play are based on the resolution of subjective symptoms. Thus, there is a great need to develop objective tools to diagnose and monitor concussion.

Several studies have evaluated biomarkers of neuronal and astroglial injury in serum of individuals (including athletes) with mild traumatic brain injury (Zetterberg et al. 2009), (Graham et al. 2011), Mannix et al. 2014). Recently, using a novel ultra-sensitive method to measure tau in plasma we found increased levels of plasma total-tau in concussed professional ice hockey players, where the levels correlated with the resolution of post-concussive symptoms and the players returning to play (Shahim et al. 2014).

Fragmentation of tau protein as a function of neuronal injury has been described in several papers (Rissman et al. 2004;Knoblach et al. 2002;Behl 2000). Recently, tau protein cleaved by ADAM10 measured in serum correlated with dementia symptoms in a Alzheimer disease (AD) patients (Henriksen et al. 2013). Another potential biomarker for neurological injury is the caspase-3 generated fragment of tau called tau-C, which reflect neuronal apoptosis (Rissman et al. 2004;Knoblach et al. 2002;Behl 2000). Recently tau-C was shown to be elevated in serum of AD patients (Henriksen et al. 2014). In this study, we tested the specific hypothesis that concussion would result in increased serum levels of tau fragments. 


\section{Methods}

\section{Study design and study population}

Serum samples were acquired from an on-going prospective multicenter cohort study of concussion among professional ice hockey players from the Swedish Hockey League (Shahim et al JAM N 2014). The study was approved by the Ethics Committee for Medical Research at the University of Gothenburg, Sweden, and the Swedish Hockey League. Written informed consent was obtained from all 288 study participants comprised of 24 players from each of the 12 teams. The teams' physicians were present during all season games and documented all signs and symptoms of concussion and recorded the date when the player had completely recovered from his concussion and was able to return to unrestricted competition. The diagnosis of concussion was based on the latest diagnostic guidelines (Barr and McCrea 2001;McCrea et al. 2003). The teams' physicians received injury protocol, the Rivermead Post-concussion Symptoms Questionnaire (Cifu et al. 2014), instructions for blood sampling, standardized blood sampling equipment, and instructions for the handling of the blood samples for the local laboratory. Prior to start of the season, all players were examined physically and with the Standardized Assessment of Concussion (Barr and McCrea 2001; McCrea et al. 2003). Players from two of the contesting teams were sampled for baseline values prior to the start of the season $(n=47)$. In addition one of these teams were also sampled prior, 1 hour and 12 hours after a friendly game without incidents of concussion. In players who sustained head injury or concussion during the season, consecutive blood samples were collected at 1 hour, 12 hours, 36 hours, and 144 hours after the injury. Players with postconcussive symptoms longer than on the 6 days were sampled on the morning of the day when the player was considered ready to return to play.

\section{Biochemical procedure}

Blood samples were collected by venipuncture into gel-separator tubes and centrifuged within 2060 minutes. Samples were aliquoted and stored at $-80^{\circ} \mathrm{C}$ for later analysis.

The neo-epitope fragments of tau degradation were assessed by solid phase competitive enzyme linked immunosorbent assays (ELISAs). Each neo-epitope constitutes a specific amino acid sequence that has been generated by enzymatic cleavage. The assays used throughout this study are based on mouse monoclonal antibodies and are specific for caspase-3 and ADAM10-generated neoepitope fragments of Tau protein, and the Tau-A assay has previously been described in detail elsewhere. ${ }^{15}$ The antibodies only react specifically with the cleaved protein and not with intact (fulllength) Tau protein, and therefore only detect Tau protein fragments.

Samples were assayed for the content of Tau-A and Tau-C using the lower limit of quantification determined to $11.9 \mathrm{ng} / \mathrm{mL}$ and $10.4 \mathrm{ng} / \mathrm{mL}$, respectively. If measured values fall below the LLOQ, the LLOQ value was assigned to the sample measurement. In brief both assays are competitive ELISA assays. The Tau-A assay detects a neoepitope identified by LC-MS/MS as the amino acid sequence TPRGAAPPGQ and the Tau-C assay detects the caspase- 3 generated cleavage site of Tau at Asp421 (Henriksen et al. 2013;Henriksen et al. 2014).

\section{Statistical analysis}

We conducted non-parametric analyses as the data was positively skewed. Data are presented as median \pm interquartile range (IQR). For the group comparison of post-concussion samples versus all preseason values (paired data not available), the Mann-Whitney $U$ test was used. For multiple group comparisons, the Kruskal-Wallis test was used. Linear regression analysis was conducted to examine whether the biomarker levels at 1 hour post-concussion could predict return to play. The area under the receiver operating characteristic curve was calculated to determine the diagnostic 
accuracy of the biomarkers. Statistical significance was determined at $p<0.05$. All statistical analyses were performed using GraphPad Prism version 6.0 (GraphPad Inc., San Diego, CA). 


\section{Results}

Of a total of 288 ice hockey players, 35 sustained sports-related concussion between September 13, 2012 and January 31, 2013, of whom 28 consented to participate in the study. The remaining concussed players either declined to participate, or had uncertain diagnosis of concussion. Three of the included players were unconscious and 25 did not lose consciousness but experienced symptoms such as headache, confusion, dizziness or nausea. The majority of the players were free of symptoms within 10 days after concussion $(n=22)$, while 6 players had post-concussive symptoms longer than 10 days.

There were no significant changes $(\mathrm{p}=0.90)$ between the serum levels of Tau-A after concussion (all time point) versus preseason samples (Figure 1A). However, the levels of Tau-C were significantly $(\mathrm{p}=0.03)$ increased in postconcussion samples compared with preseason samples (Figure 1B).

When players were followed longitudinally, the levels of Tau-A peaked at 12 hours postconcussion, ,however the increase was not statistically significant from the levels at 1 hour, 12 hours, 36 hours 144 hours or when the players returned to play, as well as compared to the preseason values (Figure 2A). Similar to Tau-A, the levels of Tau-C remained unchanged over time (Figure 2B).

\section{Severity of concussion}

As most concussion symptoms resolve within a week, we graded the severity of concussion based on the resolution of post-concussive symptom and player returning to play: 1) those who were symptom-free and hade safe return to play within 10 days, and 2) those who required return to play more than 10 days. Serum Tau-A levels at 1 hour and 12 hour post-concussion were significantly elevated in players with persistent post-concussive symptoms lasting longer than 10 days compared to players with symptoms resolving within 10 days (Figure 3A). In contrast to Tau-A, the levels of Tau-C did not differ between the players with post-concussive symptoms lasting longer than 10 days versus players with symptoms resolving withing 10 days (Figure 3B). Furthermore, serum TauA concentrations 1 hour and 12 hours after concussion correlated with the number of days it took for players to return to play (Figure 3D). However, serum Tau-C concentrations at 1 hour and 12 hours after concussion did not significantly relate to the resolution of post-concussive symptoms (Figure 3D). We also tested if there was any correlation between the levels of tau fragments and total-tau (T-tau) previously measured in the same cohort of ice hockey players (Shahim et al, JAMA $\mathrm{N}$ 2014). As shown in Figure 3E, the mean serum Tau-A levels at 1-12 hours post-concussion were linearly related to plasma T-tau levels assessed 1-12 hours post-concussion. The relationship between serum Tau-A and T-tau was less strong when either marker was sampled at other time points after concussion (data not shown). In contrast to Tau-A, there was no relationship between Tau-C levels and T-tau (Figure 3E).

\section{Diagnostic accuracy of the biomarkers}

To determine whether Tau-A and Tau-C could discriminate between players with late and early return to play, we calculated the area under the receiver operating curve (AUC). The AUC for Tau$\mathrm{A}$ at $12 \mathrm{~h}(\mathrm{AUC}=0.91 ; 95 \% \mathrm{CI}, 0.78-1, \mathrm{p}=0.005)$ was greater than at 1 hour post-concussion $(\mathrm{AUC}=0.87 ; 95 \% \mathrm{CI}, 0.71-1, \mathrm{p}=0.01)$. Tau-C was unable to discriminate between early and late return to play at both 1 hour and 12 hours post-concussion $(\mathrm{p}=0.55$ and 0.50 , respectively) (Figure $5)$. 


\section{Discussion}

The major findings of this study were that serum Tau-A concentrations at 1 and 12 hours could predict the number of days it took for the players to return to play and serum Tau-A levels at 1 hour and 12 hours after concussion could discriminate between early and late return to play.

This is the first study to measure tau fragments in serum from concussed professional athletes. Similar to the previously reported study where we measured plasma total-tau (T-tau) (Shahim et al. 2014) in the same cohort of individuals, serum Tau-A correlated with the number of days it took to return to play. In addition, there is a linear relationship between Tau-A and T-tau. In contrast to Ttau, which can only be measured using the ultra-sensitive single molecular array technique (Shahim et al. 2014), tau fragments are measured using standard ELISA. One plausible explanation which allows quantification of tau fragments in serum are that the may easily cross the blood-brain barrier, (Wang et al. 2012).

Knowing which players are at increased risk of long-term post-concussive symptoms could be of great value in the management of sports-related concussion. Serum levels of Tau-A measured at 1 hour and 12 hours post-concussion could identify these cases of high risk players, which should be managed with a higher degree of vigilance by personal specially trained (McCrory et al. 2013). Interestingly, Tau-A provides a graded response in players with post-concussive symptoms lasting longer than 10 days with the greatest diagnostic accuracy $(\mathrm{AUC}=0.91)$ being at 12 hours after concussion. Although based on a limited number of measurements, an interesting finding in this study is that the return to play values of the biomarker did significantly drop, when compared to the earlier post-concussion values. This could indicate that despite the players are declared fit to return to play, the underlying damage may still be present.

Considering the significant separation of players with short-term from the players with persistent post-concussive symptoms it was surprising that no separation between preseason and postconcussion levels was observed for serum Tau-A. However, this is potentially explained by the large variation in post-concussion levels due to the mixture of severities of concussions. Further analysis of this finding using paired pre-season and post-concussion samples are of great interest. However, Tau-C could separate between preseason and post-concussion levels. The reason for this presently is unclear, but it is appealing to assume Tau-C being related to a milder component of the injury than Tau-A.

The main limitation of this study was the relative small sample size, which restricts the possibilities of examining biomarker levels in relation to different forms and severities of concussion. Further, we did not have access to preseason samples for all ice hockey players, which would have made it easier to evaluate the comparison between post-concussion versus preseason baseline values. Finally, although measuring axonal injury by Tau-A is an important aspect of determining the severity of concussion, concussion is a heterogeneous disorder affecting all cell types in the brain (Zetterberg et al. 2013), therefore a panel of biomarkers reflecting not only cortical damage but also astrocytic, endothelial, and microglial injury may be required in the clinical management of athletes with sports-related concussion. 
In conclusion, measurement of Tau-A separates ice hockey players with short-term post-concussive symptoms from those with prolonged symptoms and hence this marker, pending further validation, could serve as a helpful tool in deciding if and when a player is fit for return to play. 


\section{Acknowledgments}

We thank all study participants and the teams for contributing to this research.

\section{Funding}

The study was supported by grants from the Swedish Research Council, the Knut and Alice Wallenberg Foundation, Centrum för Idrottsforskning and the Emil and Maria Palm Foundation. Kim Henriksen and Thomas Linemann have received funding from the Danish Research Foundation (Den Danske Forskningsfond). Dilek Inekci is funded by the Danish Ministry for Science, Technology and Education.

\section{Disclosures}

PS: none, TL: None, DI: None, KB: none, KH: Patent on Tau biomarkers, MK: Stocks in Nordic Bioscience and patents on Tau biomarkers. 
Table 1. Concentrations of Tau-A and Tau-C 1 and 12 hours after concussion based on RTP

\begin{tabular}{|c|c|c|c|c|c|}
\hline \multirow[b]{2}{*}{ Characteristic } & \multicolumn{5}{|c|}{ Median (range) } \\
\hline & $\begin{array}{l}\text { Preseason } \\
(n=44)\end{array}$ & $\begin{array}{l}\text { Post- } \\
\text { concussion } 1 \mathrm{~h} \\
\mathrm{RTP} \leq 10 \\
(\mathrm{n}=19)\end{array}$ & $\begin{array}{l}\text { Post- } \\
\text { concussion } \\
1 \mathrm{~h} \\
\text { PCS > } 10 \\
(\mathrm{n}=5)\end{array}$ & $\begin{array}{l}\text { Post- } \\
\text { concussion } \\
12 \mathrm{~h} \\
\text { RTP } \leq 10 \\
(\mathrm{n}=18)\end{array}$ & $\begin{array}{l}\text { Post- } \\
\text { concussion } \\
12 \mathrm{~h} \\
\text { RTP > } 10 \\
(\mathrm{n}=5)\end{array}$ \\
\hline Age, $y$ & & $27.5(19-40)$ & $25.5(20-34)$ & $27.5(19-38)$ & $25.5(20-34)$ \\
\hline Tau-A, ng/mL & $\begin{array}{l}30.9 \\
(11.9- \\
90.6)\end{array}$ & $\begin{array}{l}24.7(11.9- \\
134.9)\end{array}$ & $\begin{array}{l}125.2 *(26.6- \\
468.6)\end{array}$ & $\begin{array}{l}27.98(11.9- \\
141.8)\end{array}$ & $\begin{array}{l}212.8^{*} \\
(38.6-486.3)\end{array}$ \\
\hline Tau-C, ng/mL & $\begin{array}{l}17.6 \\
(11.1- \\
46.8)\end{array}$ & $19.0(8.0-28.7)$ & $\begin{array}{l}19.9(14.1- \\
34.0)\end{array}$ & $\begin{array}{l}21.1(8.5- \\
58.5)\end{array}$ & $\begin{array}{l}19.0(9.0- \\
28.7)\end{array}$ \\
\hline
\end{tabular}

Players with RTP > 10 days have significantly higher levels (*) of Tau-A at both $1 \mathrm{~h}$ and $12 \mathrm{~h}$ postconcussion compared to players with $\mathrm{RTP} \leq 10$ days and furthermore the levels are significantly higher than preseason levels. Players with RTP $\leq 10$ days did not have significantly elevated levels of Tau-A compared to preseason. Boxes represent median and bars interquartile range. 


\section{Figures}

Figure 1. Preseason and Postconcussion Levels of Tau-fragment
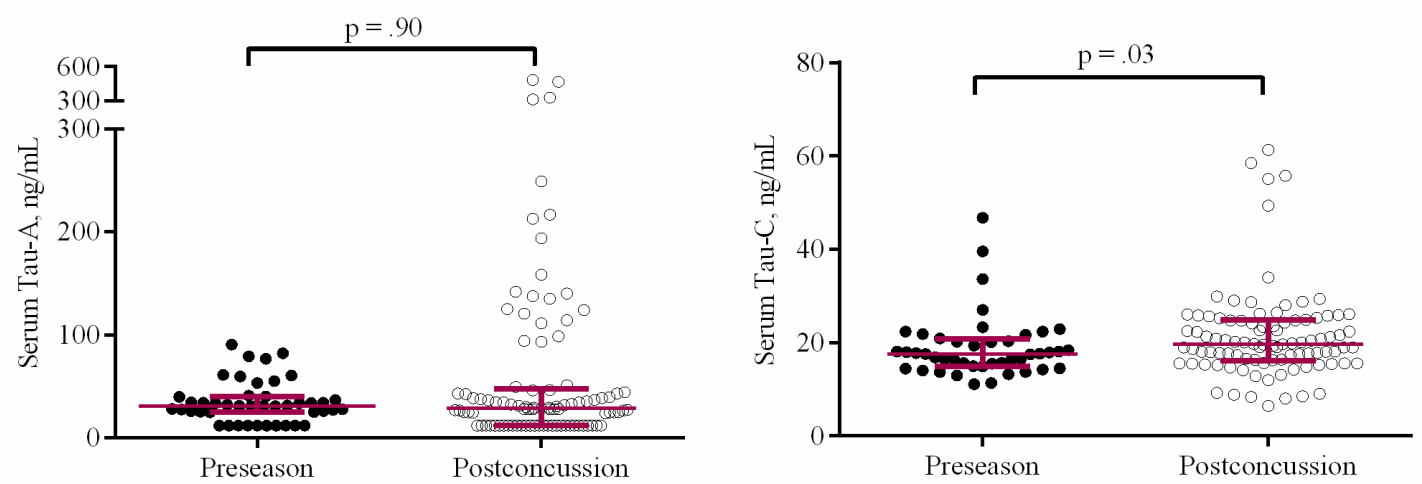

There were no significant changes between the serum levels of Tau-A after concussion (all time) versus preseason samples. However, serum Tau-C was significantly higher in postconcussion samples compared with preseason samples. Horizontal lines represent median and interquartile range. 
Figure 2. Longitudinal biomarker changes
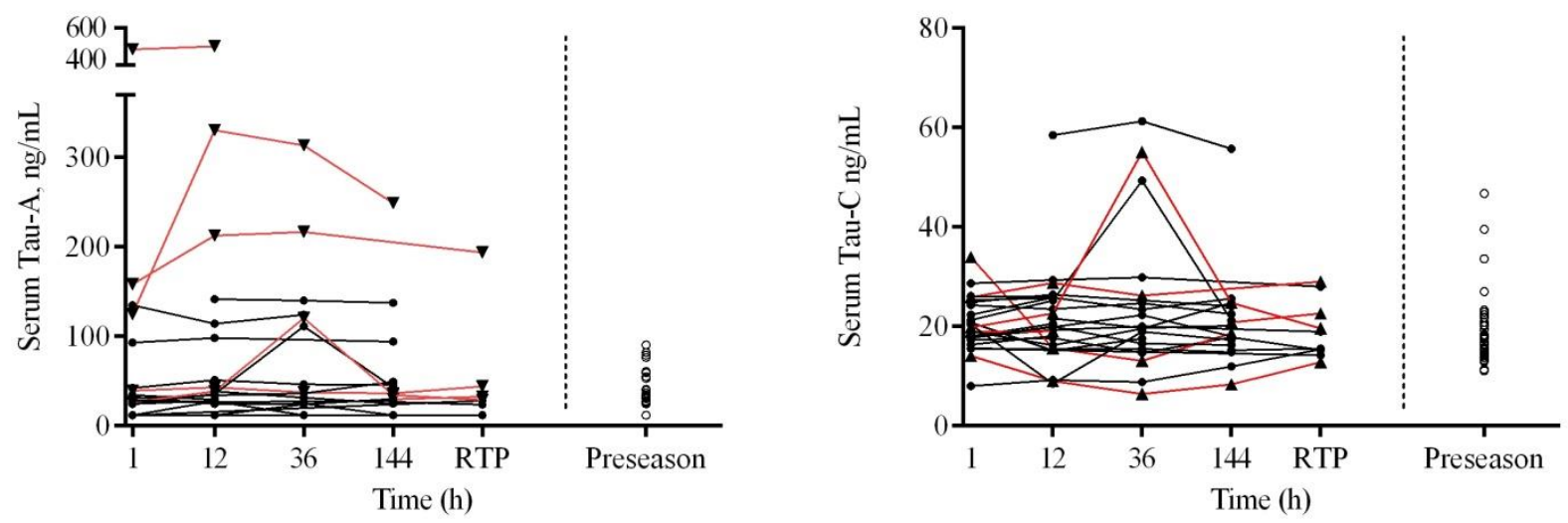

A) Median levels of Tau-A peaked at 12 hours after concussion, but were not significantly different from all other time points. B) Concentrations of Tau-C remained unchanged over time. RTP indicates return to play.

Figure 3. Biomarker concentration in relation to early versus late resolution of postconcussion symptoms
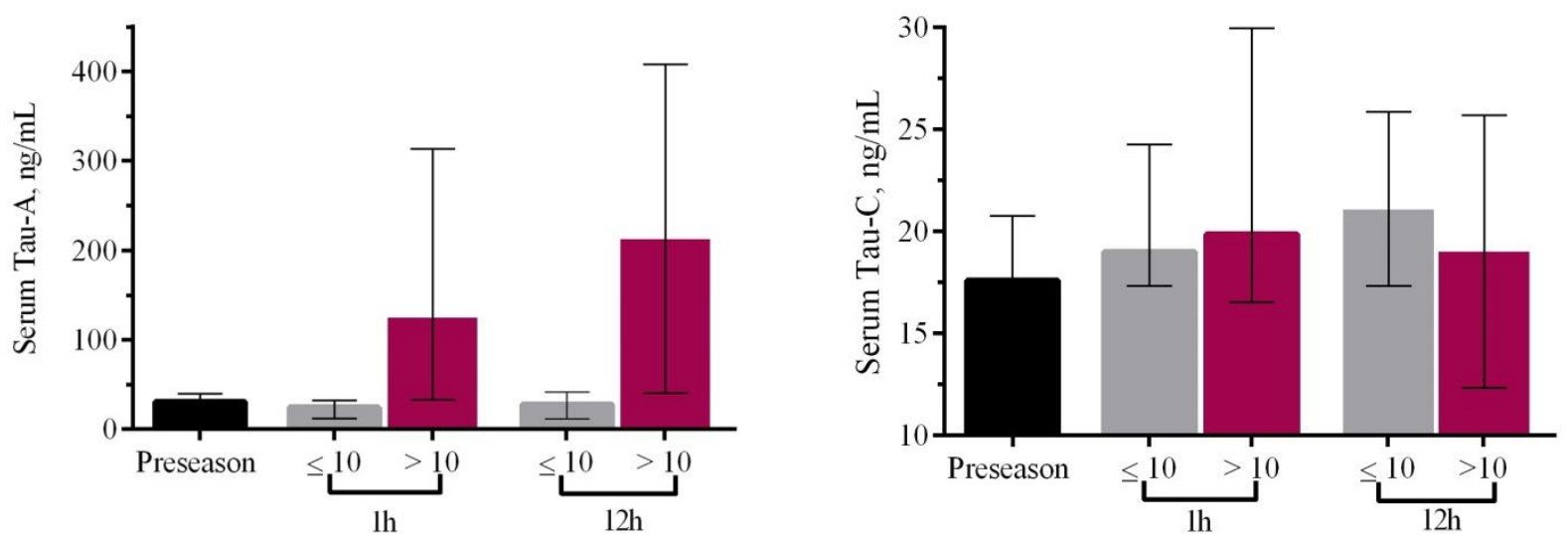

Players who had RTP > 10 days have significantly higher levels of Tau-A both $1 \mathrm{~h}$ and $12 \mathrm{~h}$ after concussion compared with players who returned to play within 10 days as well as preseason values. However, in players who RTP within 10 days, the levels of both Tau-A were not significantly elevated compared with preseason values. The levels of Tau-C did not differ significantly between different concussion categories. 
Figure 4. Serum levels of tau fragments in relation to severity of brain injury
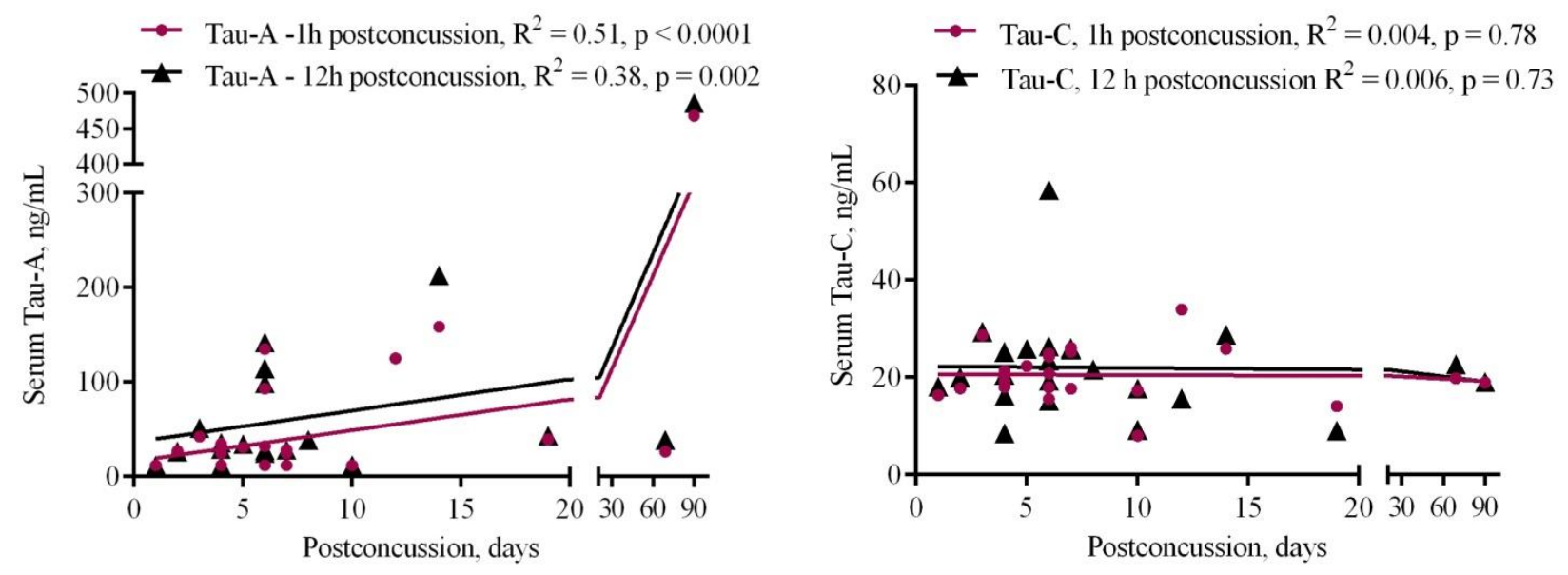

(A)Tau-A and (B) Tau-C concentrations after 1 hour and 12 hours postconcussion are shown in relation to resolution of post-concussive symptoms and return to play. One player resigned due to persistent post-concussive symptoms and was entered more than 90 days in the graph. The mean serum Tau-A concentration at 1 and 12 hours postconcussion is linearly related to the plasma tau concentration measured at 1 and 12 hour (C). For measures of either Tau-A or Tau-C and tau at all other time points the correlation between the two markers is less strong (data not shown)

Figure 5. Diagnostic accuracy of the biomarkers
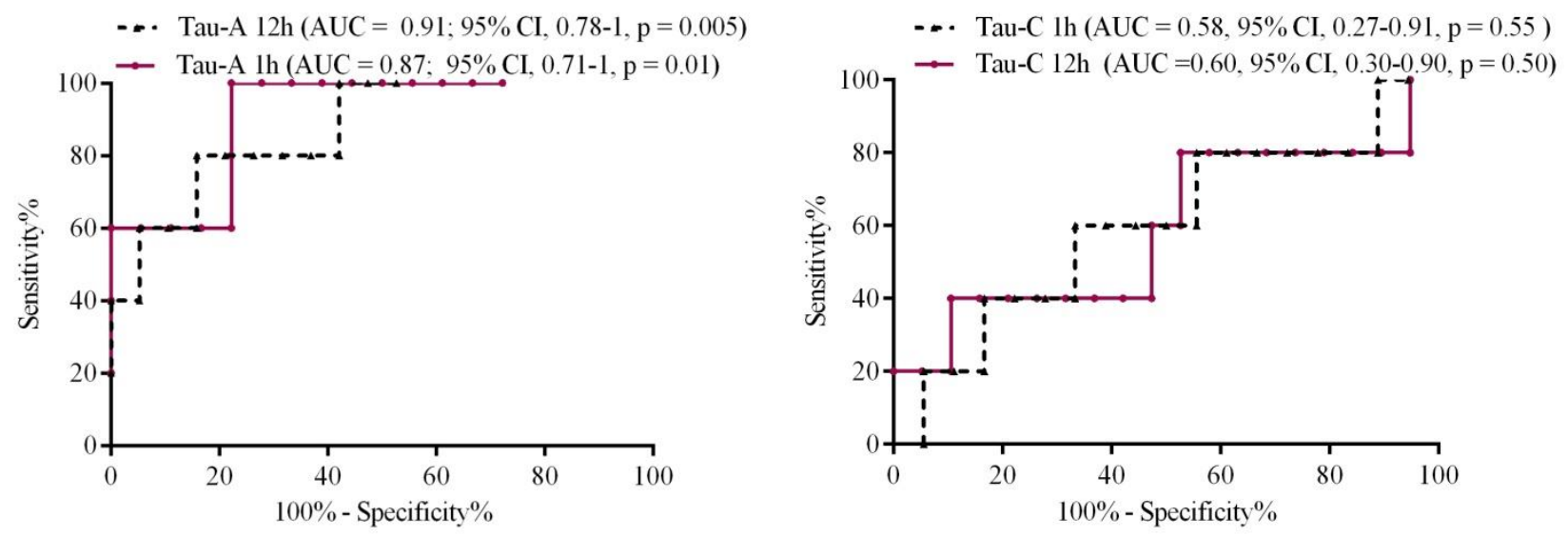
Area under the receiver operating characteristic curve (AUC) for Tau-A and Tau-C at both 1 hours and 12 hours postconcussion for players with early vs. late resolution of postconcussion symptoms. 


\section{References}

Barr W. B. and McCrea M. (2001) Sensitivity and specificity of standardized neurocognitive testing immediately following sports concussion. J. Int. Neuropsychol. Soc. 7, 693-702.

Behl C. (2000) Apoptosis and Alzheimer's disease. J. Neural Transm. 107, 1325-1344.

Cifu D. X., Walker W. C., West S. L., Hart B. B., Franke L. M., Sima A., Graham C. W. and Carne W. (2014) Hyperbaric oxygen for blast-related postconcussion syndrome: three-month outcomes. Ann. Neurol. 75, 277-286.

Graham M. R., Myers T., Evans P., Davies B., Cooper S. M., Bhattacharya K., Grace F. M. and Baker J. S. (2011) Direct hits to the head during amateur boxing is associated with a rise in serum biomarkers for brain injury. Int. J. Immunopathol. Pharmacol. 24, 119-125.

Harmon K. G., Drezner J., Gammons M., Guskiewicz K., Halstead M., Herring S., Kutcher J., Pana A., Putukian M. and Roberts W. (2013) American Medical Society for Sports Medicine position statement: concussion in sport. Clin. J. Sport Med. 23, 1-18.

Henriksen K., Byrjalsen I., Christiansen C. and Karsdal M. A. (2014) Relationship between Serum Levels of Tau Fragments and Clinical Progression of Alzheimer's Disease. J. Alzheimers. Dis. Henriksen K., Wang Y., Sorensen M. G., Barascuk N., Suhy J., Pedersen J. T., Duffin K. L., Dean R. A., Pajak M., Christiansen C., Zheng Q. and Karsdal M. A. (2013) An enzyme-generated fragment of tau measured in serum shows an inverse correlation to cognitive function. PLoS. One. 8, e64990.

Knoblach S. M., Nikolaeva M., Huang X., Fan L., Krajewski S., Reed J. C. and Faden A. I. (2002) Multiple caspases are activated after traumatic brain injury: evidence for involvement in functional outcome. J. Neurotrauma 19, 1155-1170.

Makdissi M., Darby D., Maruff P., Ugoni A., Brukner P. and McCrory P. R. (2010) Natural history of concussion in sport: markers of severity and implications for management. Am. J. Sports Med. 38, 464-471.

Mannix R., Eisenberg M., Berry M., Meehan W. P., III and Hayes R. L. (2014) Serum biomarkers predict acute symptom burden in children after concussion: a preliminary study. J. Neurotrauma 31, 1072-1075.

McCrea M., Guskiewicz K. M., Marshall S. W., Barr W., Randolph C., Cantu R. C., Onate J. A., Yang J. and Kelly J. P. (2003) Acute effects and recovery time following concussion in collegiate football players: the NCAA Concussion Study. JAMA 290, 2556-2563.

McCrory P., Meeuwisse W., Aubry M., Cantu B., Dvorak J., Echemendia R. J., Engebretsen L., Johnston K., Kutcher J. S., Raftery M., Sills A., Benson B. W., Davis G. A., Ellenbogen R. G., Guskiewicz K. M., Herring S. A., Iverson G., Jordan B. D., Kissick J., McCrea M., McIntosh A. S., Maddocks D. L., Makdissi M., Purcell L., Putukian M., Turner M., Schneider K. and Tator C. H. (2013) Consensus statement on concussion in sport--the 4th International Conference on Concussion in Sport held in Zurich, November 2012. Clin. J. Sport Med. 23, 89-117.

McCrory P., Meeuwisse W., Johnston K., Dvorak J., Aubry M., Molloy M. and Cantu R. (2009) Consensus statement on Concussion in Sport 3rd International Conference on Concussion in Sport held in Zurich, November 2008. Clin. J. Sport Med. 19, 185-200.

Mez J., Stern R. A. and McKee A. C. (2013) Chronic traumatic encephalopathy: where are we and where are we going? Curr. Neurol. Neurosci. Rep. 13, 407.

Neselius S., Zetterberg H., Blennow K., Randall J., Wilson D., Marcusson J. and Brisby H. (2013) Olympic boxing is associated with elevated levels of the neuronal protein tau in plasma. Brain Inj. 27, 425-433.

Petraglia A. L., Maroon J. C. and Bailes J. E. (2012) From the field of play to the field of combat: a review of the pharmacological management of concussion. Neurosurgery 70, 1520-1533. 
Plassman B. L., Havlik R. J., Steffens D. C., Helms M. J., Newman T. N., Drosdick D., Phillips C., Gau B. A., Welsh-Bohmer K. A., Burke J. R., Guralnik J. M. and Breitner J. C. (2000) Documented head injury in early adulthood and risk of Alzheimer's disease and other dementias. Neurology 55, 1158-1166.

Rissman R. A., Poon W. W., Blurton-Jones M., Oddo S., Torp R., Vitek M. P., LaFerla F. M., Rohn T. T. and Cotman C. W. (2004) Caspase-cleavage of tau is an early event in Alzheimer disease tangle pathology. J. Clin. Invest 114, 121-130.

Rubenstein R., Chang B., Davies P., Wagner AK M. D., Robertson C. S. and Wang K. K. (2014) A Novel, Ultrasensitive Assay for Tau: Potential for Assessing Traumatic Brain Injury in Tissues and Biofluids. J. Neurotrauma.

Shahim P., Tegner Y., Wilson D. H., Randall J., Skillback T., Pazooki D., Kallberg B., Blennow K. and Zetterberg H. (2014) Blood biomarkers for brain injury in concussed professional ice hockey players. JAMA Neurol. 71, 684-692.

Wang Y., Sorensen M. G., Zheng Q., Zhang C., Karsdal M. A. and Henriksen K. (2012) Will posttranslational modifications of brain proteins provide novel serological markers for dementias? Int. J. Alzheimers. Dis. 2012, 209409.

Zetterberg H., Smith D. H. and Blennow K. (2013) Biomarkers of mild traumatic brain injury in cerebrospinal fluid and blood. Nat. Rev. Neurol. 9, 201-210.

Zetterberg H., Tanriverdi F., Unluhizarci K., Selcuklu A., Kelestimur F. and Blennow K. (2009) Sustained release of neuron-specific enolase to serum in amateur boxers. Brain Inj. 23, 723-726. 